\title{
Three-dimensional study of planar optical antennas made of split-ring architecture outperforming dipole antennas for increased field localization
}

\author{
Veli Tayfun Kilic, Vakur B. Erturk, and Hilmi Volkan Demir* \\ Department of Electrical and Electronics Engineering, Department of Physics, and UNAM-Institute \\ of Materials Science and Nanotechnology, Bilkent University, Ankara 06800, Turkey \\ *Corresponding author: volkan@stanfordalumni.org
}

Received September 30, 2011; revised November 25, 2011; accepted November 25, 2011; posted November 28, 2011 (Doc. ID 155338); published January 9, 2012

\begin{abstract}
Optical antennas are of fundamental importance for the strongly localizing field beyond the diffraction limit. We report that planar optical antennas made of split-ring architecture are numerically found in three-dimensional simulations to outperform dipole antennas for the enhancement of localized field intensity inside their gap regions. The computational results (finite-difference time-domain) indicate that the resulting field localization, which is of the order of many thousandfold, in the case of the split-ring resonators is at least 2 times stronger than the one in the dipole antennas resonant at the same operating wavelength, while the two antenna types feature the same gap size and tip sharpness. (C) 2012 Optical Society of America

OCIS codes: $240.6680,260.5740,310.6628,260.3910$.
\end{abstract}

The spatial resolution of a conventional optical system is constrained by the diffraction limit. Beyond this limit, although transmission apertures can provide spatial resolution of the order of their aperture size in the near field, the low intensity of their transmitted fields poses a fundamental problem. With the help of plasmonic interactions between radiated electromagnetic fields and conduction electrons on metallic surfaces, optical antennas can overcome this trade-off and reach large field enhancement levels inside their gap regions beyond the diffraction limit [1]. This can help address important problems, including limited density in data storage, blurred images in microscopy, and crosstalk in detector arrays.

To date, different types of optical antennas have been investigated [2-12]. Among them, dipole and bow-tie architectures have been most extensively studied [ $\underline{3}-\underline{7}]$. Their spectral response, tuned by material and geometrical parameters, has been numerically computed [3- $\underline{-5}]$ and experimentally observed [4-7]. These antennas rely on sharp tips to obtain strong field localization. Also, nanoantennas of different shapes, such as spherical $[9,10]$ and elliptical [을 10$]$ structures, have been reported. Unfortunately, the field enhancement obtained in these antennas has not reached the largest possible levels. More complex architectures, for example, three-dimensionally V-shaped structures [11], have been shown to lead to higher enhancement levels. However, they are unfavorably harder to fabricate. Therefore, there is a strong need to enable high field enhancement levels, while avoiding the need for relying on very sharp tips or threedimensional (3D) construction.

To address this problem, in this Letter, we numerically study and demonstrate planar optical antennas that exhibit substantially increased field localization in split-ring architectures compared to those of single dipole antennas with the gaps and tips of the same size. Evolving from a starting dipole antenna by connecting its end points into a split-ring antenna, we find in 3D simulations that the field radiations from the end points are reduced, which enables a stronger localized field in the gap without decreasing the gap size or increasing the tip sharpness. This can be explained by the increased quality factor $(Q$ factor) of the connected optical antenna by transforming from the dipole into the split-ring resonator (SRR) structure. Although, previously, various forms and variants of optical SRRs have been widely studied as metamaterials for a number of interesting properties, including negative refraction and cloaking [12], there is no prior report, to the best of our knowledge, that focuses on the property of field localization capability or that analyzes and compares the field enhancement performance of SRR antennas altered from dipole antennas as a function of their 3D geometrical parameters. Therefore, this work provides a systematic comparative study for the field localization of SRR antennas, which enable a larger field enhancement than both the dipole and bow-tie antennas reported in the previous literature [3].

In the analysis, we compute the field intensity enhancement inside the gap regions of the optical antennas made of SRRs and single dipoles in the spectral range of 400 to $4000 \mathrm{~nm}$ using the finite-difference time-domain (FDTD) method (Lumerical Solutions Inc., Canada). The normalized field intensity enhancement averaged over the gap volume $V$ is calculated by

$$
\text { field enhancement }=\frac{1}{V} \int_{V}\left|\frac{E(x, y, z)}{E_{\text {inc }}}\right|^{2} \mathrm{~d} V
$$

where $E(x, y, z)$ and $E_{\text {inc }}$ are the field inside the gap region and the incident (source) field, respectively. In 3D simulations, we illuminate the antennas in the positive $z$ direction through the substrate with a plane wave polarized along the $x$ direction [see Figs. 1(a) and (b)]. A part of the computational domain that includes the antenna and additional volume surrounding the antenna (extended by 100,100 , and $25 \mathrm{~nm}$ from the antenna in the $x, y$, and $z$ directions, respectively) is meshed uniformly with a mesh size of $2.5 \mathrm{~nm}$, which was carefully chosen to mesh the corners without overspilling and to obtain good 


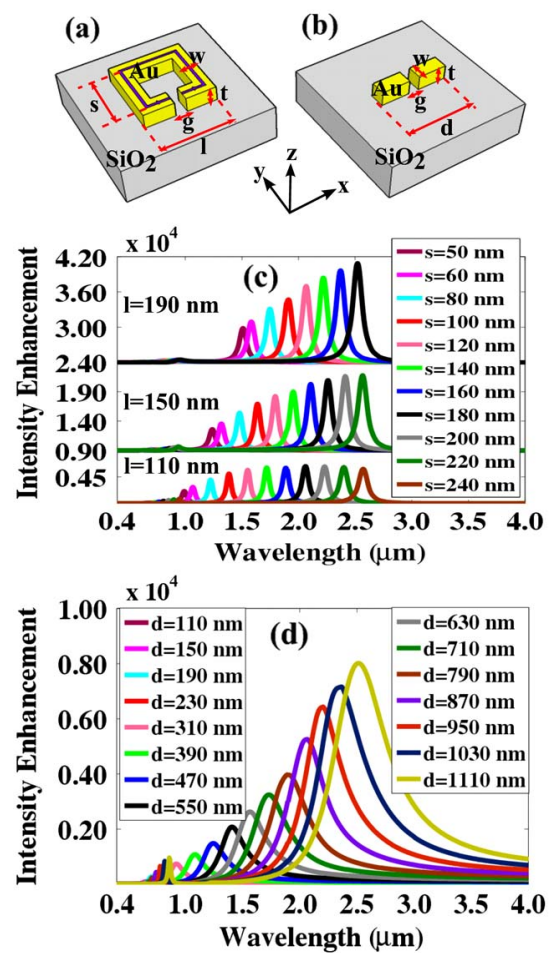

Fig. 1. (Color online) Optical antenna architecture of (a) the SRR and (b) the single dipole structure, and computed field intensity enhancement profiles (c) for the SRR and (d) the dipole. In (c), the results of the SRR for $l=110,150$. and $190 \mathrm{~nm}$ are represented starting from bottom to top, respectively, with their zero levels shifted for clarity. Also, in (c) and (d), the intensity enhancements are ordered with the dimensions $s$ and $d$, respectively, from the shorter wavelengths toward the longer.

convergence results. The rest of the computational domain is meshed using a coarser mesh size. It is truncated with perfectly matched layers.

We present the field enhancement performance of both the SRR and single dipole antennas in Fig. 1. Here the idea is to increase the field localization inside the gap region through connecting the dipole end points into the split-ring antenna and decreasing the field radiation from these end points. This connection allows the induced current to flow continuously from one gap side to the other over the SRR antenna when on resonance. This connectedness provides a new means of increasing the $Q$ factor. In the numerical simulations, silica $\left(\mathrm{SiO}_{2}\right)$ [13] is used as the dielectric platform on which the antennas are patterned because silica is commonly available, cheap, and optically transparent over a wide spectrum, including the visible and near-infrared regions. Here the metal is selected as gold [14] because of its high melting temperature, which is important for preventing the effects of high field intensity. In addition, the geometrical parameters of the SRR and dipole antennas are kept identical to avoid an unfair comparison due to the lightning rod effect $[6,10]$. The gap size $(g)$, the metal width $(w)$, and the metal thickness $(t)$ are set to be 30,40 , and $40 \mathrm{~nm}$, respectively, since patterns with these features can be realized using currently available nanofabrication processes.

We first study the field intensity enhancement behavior of the SRR architecture as a function of the antenna dimensions $l$ and $s$ in the respective $x$ and $y$ directions. The minimum sizes $\left(l_{\min }\right.$ and $\left.s_{\min }\right)$ are set to be 110 and $50 \mathrm{~nm}$, respectively, because of the constant metal width $(w)$ and the gap length $(g)$. The geometry of a single SRR antenna with $l_{\min }=110 \mathrm{~nm}$ turns into a $\mathrm{U}$ shape and $s_{\min }$ is taken to be longer than $w(40 \mathrm{~nm})$ to obtain a ring shape. On the other hand, the single dipole antenna with the varying length $d$ from 110 to $1110 \mathrm{~nm}$ is simulated for comparison purposes.

In Figs. 1(c) and (d), the resultant field enhancements inside the gap regions of the SRR and dipole antennas are presented, respectively. Figure 1(c) shows that the peak value of the field enhancement for an SRR antenna increases with the size of the antenna both along the $x$ and $y$ directions. These maximum field enhancement dependencies on the $l$ and $s$ geometrical parameters are explicitly shown in Fig. 2(a). Figure 1(c) also demonstrates that the resonance wavelength shifts toward longer wavelengths as the geometry of the antenna enlarges. Figure 2(b) further illustrates the resonance wavelength shift with respect to the SRR current path length [shown on the gold antenna in Fig. 1(a)]. Here the averaged current path length is calculated by

$$
L=(l-w-g)+(l-w)+2 s,
$$

where $w$ and $g$ are constant ( 40 and $30 \mathrm{~nm}$, respectively). Computational results show that the resonance wavelength increases almost linearly with the current path length for all cases (i.e., for $l=110,150$, and $190 \mathrm{~nm}$ ) [Fig. 2(b)]. However, the resonance wavelength is shorter for the horizontally large resonator antennas than the horizontally small ones, which have the same current path lengths.

For comparison purposes, we also study the intensity enhancement of a single dipole antenna with a varied length as presented in Fig. 1(d). We observe that the peak value of the field enhancement scales up with the length of the dipole in a similar fashion with that of the SRR antenna (Fig. 3). In addition, it is deduced from Fig. 1(d) that the resonance wavelength increases linearly with
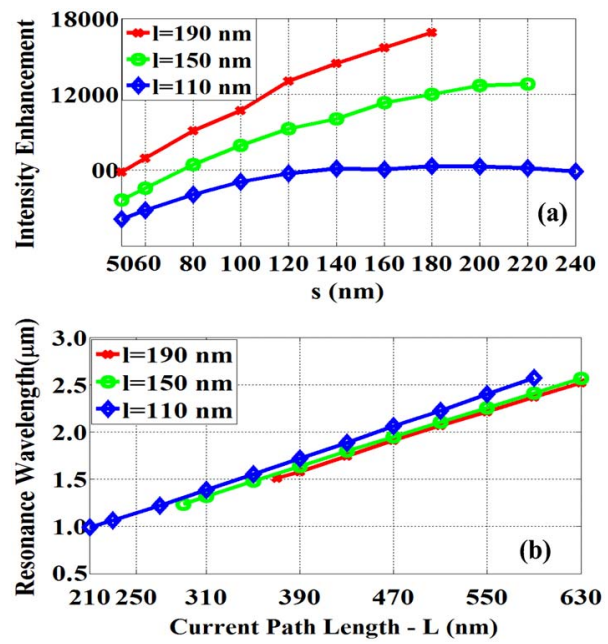

Fig. 2. (Color online) (a) Maximum field enhancement dependency on antenna geometries and (b) resonance wavelength shift with the current path length $(L)$ in the SRR antenna architecture. 


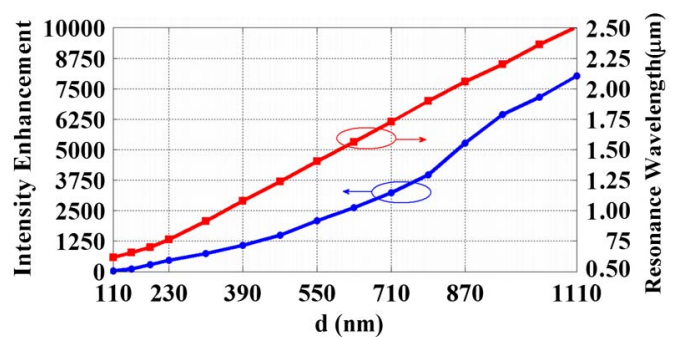

Fig. 3. (Color online) Maximum field enhancement and resonance wavelength shifts as a function of the dipole antenna length $d$. (Arrows point to the corresponding axes of the curves.)

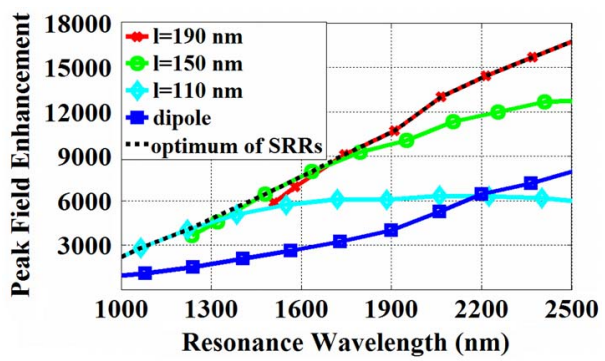

Fig. 4. (Color online) Maximum field enhancement versus resonance wavelength for single dipole and SRR antennas.

the dipole length, as depicted in Fig. 3 . However, Figs. 2 and $\underline{3}$ show that the resonance wavelength of the dipole antenna is shorter than that of the SRR antenna, even though the dipole antenna length and the SRR current path length are the same.

Furthermore, the maximum field enhancements of the SRR and dipole antennas are given as a function of their resonance wavelengths in Fig. 4 . The black dotted curve in the figure indicates the optimum field enhancements using the SRR designs with different lengths in the $x$ direction $(l)$. The ratio of this field enhancement for the optimum SRR designs to that for the dipole antenna (represented with blue curve in Fig. 4) is calculated and shown as the improvement factor in Fig. 5. This demonstrates that the resonance field intensity enhancement of a dipole nanoantenna can be increased more than 2 times with a SRR nanoantenna. This result can be well understood in terms of the $Q$ factor of the resonator. In general, it is known that the increased $Q$ factor enhances the energy storage. It is clear from Fig. 1 that the SRR antenna yields a higher $Q$ factor than the dipole. As confirmed by the field maps, this is attributed to the reduced radiation from the end points when they are connected. Also, this is supported by the current density maps that show continuous induced current flow from one gap side to the other over the split-ring antenna. This leads to sharper resonances with increased $Q$ factor. Another way of explaining higher $Q$ factor is through the role of stored magnetic energy, which was previously reported for a twodimensional geometry [15]. Therefore, this approach of connectedness introduces an alternative means of substantially enhancing the $Q$ factor and field localization to making sharper tips and smaller gaps.

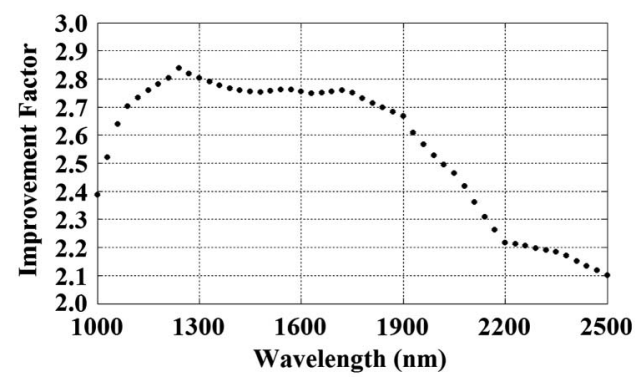

Fig. 5. Improvement factor versus wavelength for the SRR antenna with respect to the single dipole antenna.

In conclusion, we study planar optical antennas based on SRRs that outperform dipole antennas for localized field intensity inside the gap region. We numerically demonstrate that the field intensity localization of a dipole antenna can be at least doubled by using the split-ring architecture with the same gap size and tip sharpness. In addition, similar to the case of a dipole antenna, we show that the resonance wavelength of the split-ring antenna exhibits a linear dependency on the antenna dimensions. Also, we present the peak value of the field enhancement contingent upon the antenna size in the split-ring designs. We believe that this simple design strategy can be beneficial for future optical antenna applications that call for increased field localization.

We acknowledge financial support from European Science Foundation (ESF) EURYI, EC-FP7 N4E NoE, TUBA, and TUBITAK 109E002, 109E004, 110E010, and $110 \mathrm{E} 217$.

\section{References}

1. L. Novotny and N. van Hulst, Nat. Photon. 5, 83 (2011).

2. K. B. Crozier, A. Sundaramurthy, G. S. Kino, and C. F. Quate, J. Appl. Phys. 94, 4632 (2003).

3. H. Fischer and O. J. F. Martin, Opt. Express 16, 9144 (2008).

4. P. Muhlschlegel, H.-J. Eisler, O. J. F. Martin, B. Hecht, and D. W. Pohl, Science 308, 1607 (2005).

5. J. Merlein, M. Kahl, A. Zuschlag, A. Sell, A. Halm, J. Boneberg, P. Leiderer, A. Leitenstorfer, and R. Bratschitsch, Nat. Photon. 2, 230 (2008).

6. D. P. Fromm, A. Sundaramurthy, P. J. Schuck, G. Kino, and W. E. Moerner, Nano Lett. 4, 957 (2004).

7. M. D. Wissert, A. W. Schell, K. S. Ilin, M. Siegel, and H.-J. Eisler, Nanotechnology 20, 425203 (2009).

8. R. M. Bakker, A. Boltasseva, Z. Liu, R. H. Pedersen, S. Gresillon, A. V. Kildishev, V. P. Drachev, and V. M. Shalaev, Opt. Express 15, 13682 (2007).

9. L. Rogobete, F. Kaminski, M. Agio, and V. Sandoghdar, Opt. Lett. 32, 1623 (2007).

10. Y. C. Martin, H. F. Hamann, and H. K. Wickramasinghe, J. Appl. Phys. 89, 5774 (2001).

11. J. Yang, J. Zhang, X. Wu, and Q. Gong, Opt. Express 15, 16852 (2007).

12. S. D. Liu, Z. S. Zhang, and Q. Q. Wang, Opt. Express 17, 2906 (2009).

13. E. D. Palik, Handbook of Optical Constants of Solids (Academic, 1985).

14. David R. Lide, CRC Handbook of Chemistry and Physics (CRC Press, 2004).

15. G. D. Walle, T. Søndergaard, and S. I. Bozhevolnyi, Phys. Rev. B 80, 235405 (2009). 